\title{
ATLAS measurements of CP Violation and Rare decays in Beauty mesons
}

\author{
Iskander Ibragimov* on behalf of the ATLAS collaboration ${ }^{\dagger}$ \\ Department of Physics, University of Siegen, Walter-Flex-Str. 3, 57068 Siegen, Germany \\ E-mail: ibragimovahep.physik.uni-siegen.de
}

In the beauty sector ATLAS performs searches for New Physics (NP) effects by precisely measuring CP-violating parameters in the $B_{s}^{0} \rightarrow J / \psi \phi$ decay, branching fractions of very rare decays like $B_{(s)}^{0} \rightarrow \mu^{+} \mu^{-}$and angular distributions in rare processes like $b \rightarrow s \mu^{+} \mu^{-}$. This report summarises results from the angular analysis of the $B^{0} \rightarrow K^{*} \mu^{+} \mu^{-}$decay performed with Run 1 data and projections for the $B_{s}^{0} \rightarrow J / \psi \phi$ and $B_{(s)}^{0} \rightarrow \mu^{+} \mu^{-}$measurements for the Run 2 and for the High-Luminosity LHC (HL-LHC). The projections are based on extrapolations of the Run 1 results with the expected statistics corresponding to assumed $130 \mathrm{fb}^{-1}\left(3 \mathrm{ab}^{-1}\right)$ of delivered luminosity for the Run 2 (HL-LHC) and on simulations of the expected layout of a new all-silicon Inner Tracker (ITk) for the HL-LHC.

The 39th International Conference on High Energy Physics (ICHEP2018)

4-11 July 2018

Seoul, Korea

* Speaker.

${ }^{\dagger}$ This work was supported in part by grants of the German Federal Ministry of Education and Research (BMBF). 


\section{Angular analysis of the $B^{0} \rightarrow K^{*} \mu^{+} \mu^{-}$decay}

The measurement [1] is performed using $20.3 \mathrm{fb}^{-1}$ of $p p$ collision data at a centre-of-mass energy $\sqrt{s}=8 \mathrm{TeV}$ collected in 2012 by the ATLAS experiment [2]. The decay $B^{0} \rightarrow K^{*} \mu^{+} \mu^{-}$, where $K^{*} \rightarrow K^{+} \pi^{-}$, has a complex angular structure, fully described by three angles and the dimuon invariant mass squared $q^{2}$. The decay provides many observables, which can be sensitive to different types of NP. The LHCb collaboration reported a $3.4 \sigma$ deviation from the SM calculations in this decay [3]. Therefore the LHCb methodology and definitions of angular observables [4], designed to minimise uncertainties from hadronic form factors, are adopted. Similarly, the same set of trigonometric transformations of angular variables is employed in our analysis due to limited statistics.

The selected data sample consists of 787 events in the signal $q^{2}$ range of $[0.04,6.0] \mathrm{GeV}^{2}$. An extended unbinned maximum-likelihood fit of the angular distribution of the decay is performed in order to extract the angular parameters in six bins of $q^{2}$. The fit procedure comprises three sequential stages: 1) extraction of $B^{0}$ mass peak parameters from the control region data $\left(B^{0} \rightarrow\right.$ $J / \psi K^{*}$ decay); 2) extraction of signal and background shapes and yields from the fit to $m_{K \pi \mu \mu}$ distributions; 3 ) inclusion of angular variables into the fit to extract the observables. The procedure is extensively validated using pseudo-experiments. A total of 342 signal events is found by the fit.

Background studies are performed using inclusive samples of $b \bar{b} \rightarrow \mu^{+} \mu^{-} X$ and $c \bar{c} \rightarrow \mu^{+} \mu^{-} X$ decays and eleven exclusive $B^{0}, B_{s}^{0}, B^{+}$and $\Lambda_{B}$ samples. Two additional distinct background contributions are observed peaking in the distributions of angular variables in data. Their effects are assessed as systematic uncertainties by excluding the affected part of the distributions or the events around $B^{+}$and $D^{0} / D_{(s)}^{+}$masses from the fit. The largest contribution to systematics comes from fake $K^{*}$ candidates. Overall, the statistical uncertainty dominates.

The comparison of the results to the theoretical predictions of Jäger and Camalich (JC) [5], Descotes-Genon et al. (DHMV) [6] and Ciuchini et al. (CFFMPSV) [7] is shown in Fig. 1 only for the $P^{\prime}$ parameters with observed deviations between theory and our measurement; the results from LHCb [3], Belle [8] and CMS [9] are also overlaid. The $P_{4}^{\prime}$ and $P_{5}^{\prime}$ measurements in the $q^{2} \in$ $[4.0,6.0] \mathrm{GeV}^{2}$ bin are $\sim 2.7 \sigma$ away from the DHMV model. The LHCb Collaboration observed a similar deviation for $P_{5}^{\prime}$ [3]. All measurements are found to be within $3 \sigma$ of the range covered by the different predictions and also compatible with the results of the other experiments.
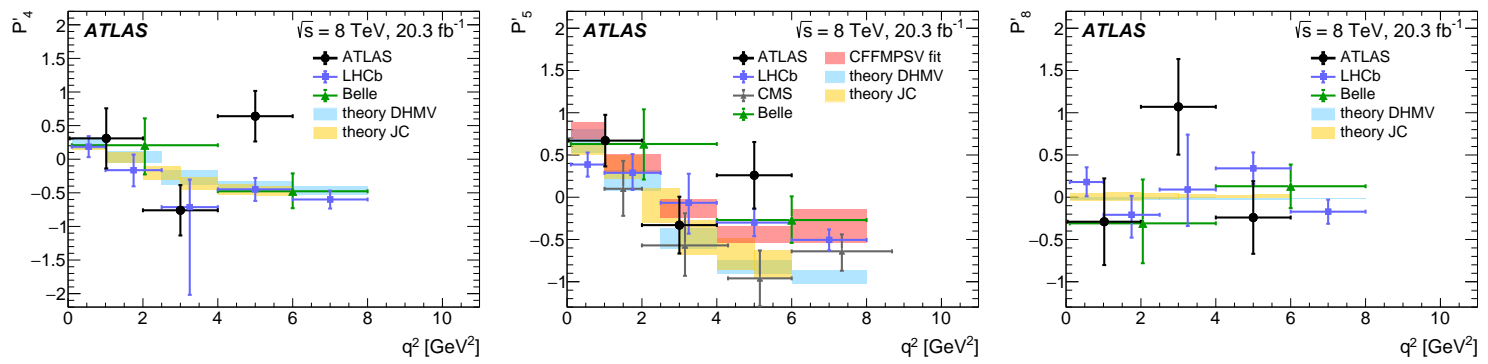

Figure 1 - The measured values of (from left to right) $P_{4}^{\prime}, P_{5}^{\prime}$ and $P_{8}^{\prime}$ parameters compared with other experimental results and predictions from theoretical calculations. Figures taken from Ref. [1]. 


\section{Prospects for the $B_{s}^{0} \rightarrow J / \psi \phi$ and $B_{(s)}^{0} \rightarrow \mu^{+} \mu^{-}$measurements}

The angular distributions in the decay $B_{s}^{0} \rightarrow J / \psi \phi \rightarrow \mu^{+} \mu^{-} K^{+} K^{-}$are sensitive to the CPviolating phase $\phi_{s}$. The ATLAS Run 1 result [10] of $\phi_{s}=-0.090 \pm 0.078$ (stat) \pm 0.041 (syst) rad agrees well with the SM and measurements by other experiments [11]. The sensitivity to $\phi_{S}$ depends predominantly on the proper decay time resolution $\sigma_{t}$, which is improved in Run 2 by $\sim 30 \%$ due to the addition of the Insertable B-Layer (IBL) [12]. An early Run 1 result projection study [13] explores the statistical reach for an integrated luminosity of $3 \mathrm{ab}^{-1}$ yielding $\sigma\left(\phi_{s}\right) \approx 0.022 \mathrm{rad}$. A later study [14] indicates that by the use of topological muon triggers three times more data can be collected, achieving further improvements.

The branching fraction measurement of the very rare decays $B_{(s)}^{0} \rightarrow \mu^{+} \mu^{-}$will benefit from the increased statistics and the improved $B_{(s)}^{0}$ invariant mass resolution at the HL-LHC. The ATLAS Run 1 result [15] is compatible with the SM at $\sim 2 \sigma$ level and the $\mathscr{B}\left(B_{(s)}^{0} \rightarrow \mu^{+} \mu^{-}\right)$values are lower than the CMS-LHCb combined result [16] or the more recent LHCb update [17]. A study [14] shows that the separation of the $B_{s}^{0}$ and $B^{0}$ mass peaks improves by a factor of 1.65 (1.5) to $2.3 \sigma(1.3 \sigma)$ in the barrel (end-cap) region compared to Run 1 for the HL-LHC case, mainly due to the lower material budget of the ITk. Using this result and pseudo-experiments based on the likelihood of the Run 1 analysis, another study [19] investigated the potential for measuring $\mathscr{B}\left(B_{(s)}^{0} \rightarrow \mu^{+} \mu^{-}\right)$with the expected datasets from the full LHC Run $2\left(130 \mathrm{fb}^{-1}\right)$ and the HL-LHC $\left(3 \mathrm{ab}^{-1}\right)$. Taking into account the prescales of topological dimuon triggers, the signal statistics estimate for the Run 2 scenario is 7 times the number of signal events in Run 1. For the HL-LHC case, three potential trigger scenarios are considered, yielding multiples of 15 ("conservative"), 60 ("intermediate") and 70 ("high yield") of the Run 1 statistics. The internal sources of systematic uncertainties like the fit shapes and efficiencies were scaled according to the increase in statistics whereas the external ones like on $\mathscr{B}\left(B^{ \pm} \rightarrow J / \psi K^{ \pm}\right)$and the $b$-quark fragmentation fractions $f_{s} / f_{d}$ were kept the same as in the Run 1 analysis; the latter contribution dominates the systematic uncertainty on $\mathscr{B}\left(B_{s}^{0} \rightarrow \mu^{+} \mu^{-}\right)$. The contours in Fig. 2 demonstrate the expected improvement in the accuracy of the $\mathscr{B}\left(B_{(s)}^{0} \rightarrow \mu^{+} \mu^{-}\right)$measurements for the LHC Run 2 and HL-LHC.
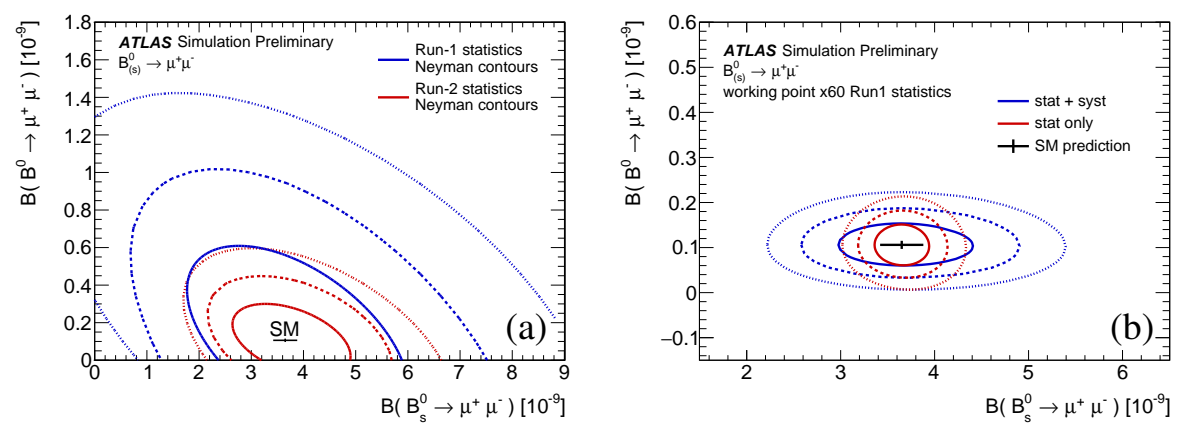

Figure 2 - Contours of $68.3 \%$ (solid), $95.5 \%$ (dashed) and $99.7 \%$ (dotted) confidence levels for $(a)$ the Run 2 projection [19] (in red) and the Run 1 statistics (in blue), obtained exploiting the 2D Neyman belt construction; $(b)$ the "intermediate" HL-LHC extrapolation scenario [19], obtained using a profiled likelihood. Red contours are statistical only; blue contours include systematics uncertainties extrapolated from the Run 1 analysis. The black points show the SM theoretical prediction and its uncertainty [18]. 


\section{References}

[1] ATLAS Collaboration, Angular analysis of $B_{d}^{0} \rightarrow K^{*} \mu^{+} \mu^{-}$decays in pp collisions at $\sqrt{s}=8 \mathrm{TeV}$ with the ATLAS detector, JHEP 10 (2018) 47, arXiv: 1805.04000 [hep-ex].

[2] ATLAS Collaboration, The ATLAS Experiment at the CERN Large Hadron Collider, JINST 3 (2008) S08003.

[3] LHCb Collaboration, Angular analysis of the $B^{0} \rightarrow K^{* 0} \mu^{+} \mu^{-}$decay using $3 \mathrm{fb}^{-1}$ of integrated luminosity, JHEP 02 (2016) 104, arXiv: 1512.04442 [hep-ex].

[4] LHCb Collaboration, Measurement of Form-Factor Independent Observables in the Decay $B^{0} \rightarrow K^{* 0} \mu^{+} \mu^{-}$, Phys. Rev. Lett. 111 (2013) 191801, arXiv: 1308.1707 [hep-ex].

[5] S. Jäger and J. Martin Camalich, Reassessing the discovery potential of the $B \rightarrow K^{*} \ell^{+} \ell^{-}$decays in the large-recoil region: SM challenges and BSM opportunities, Phys. Rev. D 93 (2016) 014028.

[6] S. Descotes-Genon et al., On the impact of power corrections in the prediction of $B \rightarrow K^{*} \mu^{+} \mu^{-}$ observables, JHEP 12 (2014) 125, arXiv: 1407.8526 [hep-ph].

[7] M. Ciuchini et al., $B \rightarrow K^{*} \ell^{+} \ell^{-}$decays at large recoil in the Standard Model: a theoretical reappraisal JHEP 06 (2016) 116, arXiv: 1512.07157 [hep-ph].

[8] Belle Collaboration, Measurement of the Differential Branching Fraction and Forward-Backward Asymmetry for $B \rightarrow K^{(*)} l^{+} l^{-}$, Phys. Rev. Lett. 103 (2009) 171801, arXiv: 0904.0770 [hep-ex].

[9] CMS Collaboration, Angular analysis of the decay $B^{0} \rightarrow K^{* 0} \mu \mu$ from pp collisions at $\sqrt{s}=8 \mathrm{TeV}$, Phys. Lett. B 753 (2016) 424, arXiv: 1507.08126 [hep-ex].

[10] ATLAS Collaboration, Measurement of the CP-violating phase $\phi_{s}$ and the $B_{s}^{0}$ meson decay width difference with $B_{S}^{0} \rightarrow J / \psi \phi$ decays in ATLAS, JHEP 08 (2016) 147.

[11] Y. Amhis et al., Averages of b-hadron, c-hadron and tau-lepton properties as of summer 2016, Eur. Phys. J. C77 (2017) 895, arXiv: 1612.07233 [hep-ph].

[12] ATLAS Collaboration, Production and Integration of the ATLAS Insertable B-Layer, 2018 JINST 13 T05008, arXiv: 1803.00844 [hep-ex].

[13] ATLAS Collaboration, ATLAS B-physics studies at increased LHC luminosity, potential for $C P$-violation measurement in the $B_{s}^{0} \rightarrow J / \psi \phi$ decay, ATL-PHYS-PUB-2013-010.

[14] ATLAS Collaboration, Expected performance for an upgraded ATLAS detector at High-Luminosity LHC, ATL-PHYS-PUB-2016-026 [https: / / cds . cern. ch/record/2223839].

[15] ATLAS Collaboration, Study of the rare decays of $B_{s}^{0}$ and $B^{0}$ into muon pairs from data collected during the LHC Runl with the ATLAS detector, Eur. Phys. J. C (2016) 76:513.

[16] CMS and LHCb Collaborations, Observation of the rare $B_{s}^{0} \rightarrow \mu^{+} \mu^{-}$decay from the combined analysis of CMS and LHCb data, Nature, 522, 2015.

[17] LHCb Collaboration, Measurement of the $B_{s}^{0} \rightarrow \mu^{+} \mu^{-}$branching fraction and effective lifetime and search for $B^{0} \rightarrow \mu^{+} \mu^{-}$decays, Phys. Rev. Lett. 118 (2017) 191801, arXiv: 1703.05747 [hep-ex].

[18] C. Bobeth et al., $B_{(s)}^{0} \rightarrow \ell^{+} \ell^{-}$in the Standard Model with Reduced Theoretical Uncertainty, Phys. Rev. Lett. 112 (2014) 101801, arXiv: 1311.0903 [hep-ph].

[19] ATLAS Collaboration, Prospects for the $\mathscr{B}\left(B_{(s)}^{0} \rightarrow \mu^{+} \mu^{-}\right)$measurements with the ATLAS detector in Run 2 LHC and HL-LHC data campaigns, ATL-PHYS-PUB-2018-005. 